\title{
Multivariate ECOGARCH processes
}

\author{
Stephan Haug, Robert Stelzer
}

\begin{abstract}
A multivariate extension of the exponential continuous time $\operatorname{GARCH}(p, q)$ model (ECOGARCH) is introduced and studied. Stationarity and mixing properties of the new stochastic volatility model are investigated and ways to model a component-wise leverage effect are presented.
\end{abstract}

\section{INTRODUCTION}

GARCH type processes have become very popular in financial econometrics to model returns of stocks, exchange rates and other series observed at equidistant time points. They have been designed (see [11] and [4]) to capture so-called stylised facts of such data, which are e.g. stochastic volatility clustering, dependence without correlation and tail heaviness. Another characteristic is that stock returns seem to be negatively correlated with changes in the volatility, i.e. that volatility tends to increase after negative shocks in the price and to fall after positive ones. This effect is called leverage effect and cannot be modelled by a GARCH type process without further extensions. This finding led [22] to introduce the exponential GARCH process, which is able to model this asymmetry. In that paper the log-volatility of the $\operatorname{EGARCH}(p, q)$ process was modelled as an $\operatorname{ARMA}(q, p-1)$ process.

Starting with [21] continuous time models related to GARCH processes have been investigated for a long time. As several important characteristic features of GARCH processes get lost in the originally studied diffusion limits of GARCH processes, [17] introduced the COG$\mathrm{ARCH}$ process as a continuous time analogue of the GARCH process, which inherits many of the characteristic features of GARCH processes. Likewise, [13] recently defined and analysed an EGARCH process in continuous time and [8] presented first estimation results.

In this paper we develop and analyse a multivariate version of the exponential continuous time GARCH process (ECOGARCH) of [13]. Note that in discrete time matrix exponential GARCH processes have for the first time been studied by [16] in a truly multivariate sense, whereas before only the variances, but not the whole covariance matrix, have been modelled as EGARCH processes (cf. [23], [31] or [32] for some typical examples).

In our EGARCH specification we model the logarithm of the covariance matrix process as a CARMA process in the symmetric matrices using the multivariate continuous time ARMA processes (CARMA) introduced in [20]. Taking the exponential then automatically ensures positive definiteness of the covariance matrix process. The standard mathematical fact that the exponential of a symmetric matrix is positive definite seems to have been used only very rarely in order to model covariance matrices so far (the recent paper [16], for instance, does not credit any references for this idea). To the best of our knowledge the first appearance in the statistics literature is [7].

AMS 2010 subject classification: Primary:60G51, 60H10; Secondary: 91B72, 91B84

Key words and phrases: CARMA process, leverage effect, Lévy process, multivariate exponential COGARCH, stochastic volatility 
One main feature of our model is the inclusion of the leverage effect. We will give some (approximate) calculations and examples which show how to choose the parameters to obtain a leverage effect. In other multivariate models in continuous time inclusion of this effect is far from easy. The multivariate Ornstein-Uhlenbeck type model of [24] looses (like in the univariate case, see [2]) much of its tractability and its pure stochastic volatility nature. In the multivariate COGARCH of [30] it seems necessary to have only positive jumps in the volatility, thus one cannot have positive shocks which lead to a lower volatility. In the multivariate variance Gamma model of [28] one does not have a volatility process and needs the multidimensional time process to be independent of the multidimensional Brownian motion. Finally, in purely Brownian motion based models, e.g. the Wishart models of [12], one can have negative dependence between volatility and price, but one can no longer speak of shocks and look at the relation between jumps in the price and in the volatility, because there are no jumps. Hence, in these models one has to quantify the leverage effect differently than we do later on.

The paper is now organised as follows. At the end of this section some notation used throughout is given. In Section 2 we first recall some basic facts on multivariate Lévy processes and on the multivariate Lévy-driven CARMA process, as defined in [20]. We further give a sufficient condition for the existence of the $\alpha$-th exponential moment of a CARMA process. In the second part of the section we introduce a general specification of the discrete time multivariate EGARCH process and propose two ways of modelling asymmetric behaviour in the vectorised log-volatility process. In the first part of Section 3 the multivariate ECOGARCH process is defined and stationarity conditions are discussed. In the second part we show the strong mixing property of the volatility and the return process and shortly consider the mean and autocovariance function of the return process. The third part provides an approximate calculation of the leverage effect. In the last part we briefly discuss a result of [29], viz. that for an ECOGARCH $(1,1)$ process there exists a sequence of $\operatorname{EGARCH}(1,1)$ processes converging to the ECOGARCH process, which adds important insight regarding the relation between our continuous time model and discrete time multivariate EGARCH processes. Finally, we present some explicit examples along with simulations in the final Section 4.

\subsection{Notation}

Throughout this paper we write $\mathbb{R}^{+}$for the positive real numbers including zero and we denote the set of real $d \times m$ matrices by $M_{d, m}(\mathbb{R})$. If $d=m$, we simply write $M_{d}(\mathbb{R})$ and denote the group of invertible $d \times d$ matrices by $G L_{d}(\mathbb{R})$, the linear subspace of symmetric matrices by $\mathrm{S}_{d}$, the (closed) positive semi-definite cone by $\mathrm{S}_{d}^{+}$and the open (in $\mathrm{S}_{d}$ ) positive definite cone by $\mathrm{S}_{d}^{++} . I_{d}$ stands for the $d \times d$ identity matrix, $\operatorname{det}(A)$ for the determinant and $\sigma(A)$ for the spectrum (the set of all eigenvalues) of a matrix $A \in M_{d}(\mathbb{R})$. Moreover, vech : $S_{d} \rightarrow \mathbb{R}^{d(d+1) / 2}$ denotes the "vector-half" operator that stacks the columns of the lower triangular part of a symmetric matrix below another. Finally, $A^{*}$ is the adjoint of a matrix $A \in M_{d}(\mathbb{R})$.

Norms of vectors and matrices are denoted by $\|\cdot\|$. If the norm is not specified then it is irrelevant which particular norm is used.

The exponential of a matrix $A$ is denoted by $\exp (A)$ or $e^{A}$ (see [14, Ch. 6] for a detailed discussion). Recall that for square matrices it is defined by functional calculus and it holds that

$$
\exp (A)=\sum_{k=0}^{\infty} \frac{A^{k}}{k !}
$$

From functional calculus it is immediately clear that the matrix exponential maps the symmetric $d \times d$ matrices to the positive definite ones. Moreover, we denote by $A^{1 / 2}$ the unique positive semi-definite square root of a matrix $A \in \mathrm{S}_{d}^{+}$. 
For a matrix $A$ we denote by $A_{i j}$ the element in the $i$-th row and $j$-th column and this notation is extended to processes in a natural way.

Regarding all random variables and processes we assume that they are defined on a given appropriate filtered probability space $\left(\Omega, \mathcal{F}, P,\left(\mathcal{F}_{t}\right)_{t \in \mathbb{R}^{+}}\right)$satisfying the usual hypotheses (complete and right continuous filtration). $L^{p}$ denotes as usual the space of all random variables with a finite $p$-th moment, i.e. all random variables $X$ with $\mathbb{E}\left(\|X\|^{p}\right)<\infty$ in a multivariate setting.

\section{THE BUILDING BLOCKS}

Before we introduce a general specification of the discrete time multivariate EGARCH process, we briefly review multivariate Lévy and CARMA processes.

\subsection{Multivariate Lévy and Lévy-driven CARMA processes}

\subsubsection{Basic facts on multivariate Lévy processes}

Now we state some elementary properties of multivariate Lévy processes that will be needed. For a more general treatment and proofs we refer to [27], [1] or [25].

We consider a Lévy process $L=\left(L_{t}\right)_{t \in \mathbb{R}^{+}}$(where $L_{0}=0$ a.s.) in $\mathbb{R}^{d}$ determined by its characteristic function $\mathbb{E}\left[e^{i\left\langle u, L_{t}\right\rangle}\right]=\exp \left\{t \psi_{L}(u)\right\}, t \geq 0$, in the Lévy-Khintchine form where

$$
\psi_{L}(u)=i\left\langle\gamma_{L}, u\right\rangle-\frac{1}{2}\left\langle u, C_{L} u\right\rangle+\int_{\mathbb{R}^{d}}\left(e^{i\langle u, x\rangle}-1-i\langle u, x\rangle \mathbb{1}_{[0,1]}(\|x\|)\right) v_{L}(d x)
$$

for $u \in \mathbb{R}^{d}, \gamma_{L} \in \mathbb{R}^{d}, C_{L} \in \mathbb{S}_{d}^{+}$and $v_{L}$ is a measure on $\left(\mathbb{R}^{d}, \mathcal{B}\left(\mathbb{R}^{d}\right)\right)$ that satisfies $v_{L}(\{0\})=0$ and $\int_{\mathbb{R}^{d}}\left(\|x\|^{2} \wedge 1\right) v_{L}(d x)<\infty$. The measure $v_{L}$ is referred to as the Lévy measure of $L$. A Lévy process is said to be a pure jump one if the Brownian part vanishes, i.e. $C_{L}=0$.

It is a well-known fact that to every càdlàg Lévy process $L$ on $\mathbb{R}^{d}$ one can associate a random measure $N_{L}$ on $\mathbb{R}^{+} \times \mathbb{R}^{d} \backslash\{0\}$ describing the jumps of $L$ (see e.g. [15, Section II.1]). For any measurable set $B \subset \mathbb{R}^{+} \times \mathbb{R}^{d} \backslash\{0\}$,

$$
N_{L}(B)=\sharp\left\{s \geq 0:\left(s, L_{s}-L_{s-}\right) \in B\right\} .
$$

The jump measure $N_{L}$ is a Poisson random measure (as defined in [15, Definition II.1.20]) on $\mathbb{R}^{+} \times \mathbb{R}^{d} \backslash\{0\}$ with intensity measure $n_{L}(d s, d x)=d s v_{L}(d x)$. By the Lévy-Itô decomposition we can rewrite $L$ almost surely as

$$
L_{t}=\gamma_{L} t+B_{t}+\int_{\|x\| \geq 1} \int_{0}^{t} x N_{L}(d s, d x)+\lim _{\varepsilon \downarrow 0} \int_{\varepsilon \leq\|x\| \leq 1} \int_{0}^{t} x \tilde{N}_{L}(d s, d x)
$$

for every $t \geq 0$. Here $B$ is a Brownian motion in $\mathbb{R}^{d}$ with covariance matrix $C_{L}, \tilde{N}_{L}(d s, d x)=$ $N_{L}(d s, d x)-d s v_{L}(d x)$ is the compensated jump measure, the terms in (2.1) are independent and the convergence in the last term is a.s. and locally uniform in $t \geq 0$.

In the sequel we will sometimes work with a two-sided Lévy process $L=\left(L_{t}\right)_{t \in \mathbb{R}}$, constructed by taking two independent copies $\left(L_{1, t}\right)_{t \in \mathbb{R}^{+}},\left(L_{2, t}\right)_{t \in \mathbb{R}^{+}}$of a one-sided Lévy process and setting

$$
L_{t}= \begin{cases}L_{1, t} & \text { if } \quad t \geq 0 \\ -L_{2,-t-} & \text { if } \quad t<0\end{cases}
$$


Assuming that $v_{L}$ satisfies additionally $\int_{\|x\|>1}\|x\|^{2} v_{L}(d x)<\infty, L$ has finite mean and covariance matrix $\Sigma_{L}$ given by $\Sigma_{L}=C_{L}+\int_{\mathbb{R}^{d}} x x^{*} v_{L}(d x)$.

For the theory of stochastic integration and SDEs (with respect to Lévy processes and/or random measures) we refer to any of the standard texts, e.g. [15], [25] or [1].

\subsubsection{Multivariate Lévy-driven CARMA processes}

As the name "continuous time ARMA" (CARMA) already suggests, these processes are the continuous time analogue of the well-known ARMA processes. A $d$-dimensional CARMA $(q, p)$ process $Y$ with $q, p \in \mathbb{N}_{0}$ can be viewed as the stationary solution to the formal differential equation:

$$
Q(\mathcal{D}) Y_{t}=P(\mathcal{D}) \mathcal{D} L_{t},
$$

where $L=\left(L_{t}\right)_{t \in \mathbb{R}}$ is a $d$-dimensional Lévy process and $\mathcal{D}$ the differential operator with respect to $t$.

$$
\begin{aligned}
& Q(z)=z^{q}+A_{1} z^{q-1}+A_{2} z^{q-2}+\ldots+A_{q} \\
& P(z)=B_{0} z^{p}+B_{1} z^{p-1}+\ldots+B_{p}
\end{aligned}
$$

with $B_{0}, \ldots, B_{p}, A_{1}, \ldots, A_{q} \in M_{d}(\mathbb{R}), A_{q} \in G L_{d}(\mathbb{R})$ and $B_{0} \neq 0$ are referred to as the autoregressive and moving average polynomial, respectively. In order to be able to define CARMA processes properly one needs $q>p$ and that the zeros of $\operatorname{det}(Q(z))$ have all strictly negative real parts. Then the $\operatorname{CARMA}(q, p)$ process $Y$ is defined as the unique stationary solution of

$$
\begin{aligned}
Y_{t} & =\left(I_{d}, 0, \ldots, 0\right) X_{t} \\
d X_{t} & =A X_{t} d t+\tilde{B} d L_{t},
\end{aligned}
$$

where

$$
A=\left(\begin{array}{ccccc}
0 & I_{d} & 0 & \cdots & 0 \\
0 & 0 & I_{d} & \cdots & 0 \\
\vdots & \vdots & \vdots & \ddots & \vdots \\
0 & 0 & 0 & \cdots & I_{d} \\
-A_{q} & -A_{q-1} & -A_{q-2} & \cdots & -A_{1}
\end{array}\right) \in M_{d q}(\mathbb{R})
$$

and $\tilde{B}=\left(\tilde{B}_{1}^{*}, \tilde{B}_{2}^{*}, \ldots, \tilde{B}_{q}^{*}\right)^{*}$ is a $q d \times d$ matrix with elements $\tilde{B}_{q-j}=-\sum_{i=1}^{q-j-1} A_{i} \tilde{B}_{q-j-i}+B_{p-j}$ for $j=0,1, \ldots, q-1$ (setting $B_{i}=0$ for $i<0$ ). The process $X$ is usually called state space representation.

Later on we need the following result on the existence of exponential moments. By Ei : $\mathbb{R} \backslash\{0\} \rightarrow \mathbb{R}$ we denote the exponential integral, i.e.

$$
\operatorname{Ei}(x)=\int_{-\infty}^{x} \frac{e^{t}}{t} d t=\gamma+\ln |x|+\sum_{k=1}^{\infty} \frac{x^{k}}{k \cdot k !} \text { for all } x \in \mathbb{R} \backslash\{0\}
$$

taking the Cauchy principal value of the integral for $x>0$ and $\gamma$ being the Euler constant.

Proposition 2.1. Let $Y$ be a stationary d-dimensional CARMA(q, $p)$ process satisfying

$$
\sigma(A) \subset(-\infty, 0)+i \mathbb{R},
$$

$\|\cdot\|$ a norm on $\mathbb{R}^{d}$ and its induced operator norm, $\alpha>0$ and $C, b>0$ such that

$$
\left\|\left(I_{d}, 0, \ldots, 0\right) e^{A s} \tilde{B}\right\| \leq C e^{-b s}
$$


for all $s \geq 0$. If

$$
\int_{\|x\| \geq 1} \operatorname{Ei}(\alpha C\|x\|) v_{L}(d x)<\infty
$$

then $\mathbb{E}\left(e^{\alpha\left\|Y_{0}\right\|}\right)<\infty$.

Let $\max (\Re(\sigma(A)))$ be the maximal real part of all eigenvalues of $A$. Then for all $0<b<-\max (\Re(\sigma(A)))$ there exists $a C>0$ such that $\left\|\left(I_{d}, 0, \ldots, 0\right) e^{A s} \tilde{B}\right\| \leq C e^{-b s}$ holds for all $s \geq 0$. If $A$ is diagonalisable this holds also for $b=-\max (\Re(\sigma(A)))$. Furthermore, (2.5) is implied by

$$
\int_{\|x\| \geq 1} e^{\alpha C\|x\|} \nu_{L}(d x)<\infty
$$

Proof. It is elementary to see (using e.g. the Jordan decomposition of $A$ ) that $\sigma(A) \subset(-\infty, 0)+$ $i \mathbb{R}$ implies for all $0<b<-\max (\Re(\sigma(A)))$ that there exists a $C>0$ such that $\left\|\left(I_{d}, 0, \ldots, 0\right) e^{A s} \tilde{B}\right\| \leq C e^{-b s}$ holds for all $s \geq 0$. If $A$ is diagonalisable, this also shows that one can take $b=-\max (\Re(\sigma(A)))$.

From Proposition 3.27 of [20] we know that the stationary distribution of $Y$ is infinitely divisible. Denote by $\left(\gamma_{Y}, \sigma_{Y}, v_{Y}\right)$ the characteristic triplet of the stationary distribution of $Y$. [27, Theorem 25.3] implies that for all $\alpha>0$ we have $\mathbb{E}\left(e^{\alpha\left\|Y_{1}\right\|}\right)<\infty$ if and only if

$$
\int_{\|x\| \geq 1} e^{\alpha\|x\|} V_{Y}(d x)<\infty
$$

Proposition 3.27 of [20] implies

$$
\begin{aligned}
\int_{\|x\| \geq 1} e^{\alpha\|x\|} \nu_{Y}(d x) & =\int_{0}^{\infty} \int_{\mathbb{R}^{d}} e^{\alpha\left\|\left(I_{d}, 0, \ldots, 0\right) e^{A s} \tilde{B} x\right\|_{1}} \mathbb{1}_{[1, \infty)}\left(\left\|\left(I_{d}, 0, \ldots, 0\right) e^{A s} \tilde{B} x\right\|\right) v_{L}(d x) d s \\
& \leq \int_{0}^{\infty} \int_{\mathbb{R}^{d}} e^{\alpha C e^{-b s}\|x\|} \mathbb{1}_{[1, \infty)}\left(\alpha C e^{-b s}\|x\|\right) v_{L}(d x) d s \\
& =\int_{\|x\| \geq 1 /(\alpha C)} \int_{0}^{\ln (\alpha C\|x\|) / b} e^{\alpha C e^{-b s}\|x\|} d s v_{L}(d x) \\
& =\frac{1}{b} \int_{\|x\| \geq 1 /(\alpha C)} \int_{1}^{\alpha C\|x\|} \frac{e^{z}}{z} d z v_{L}(d x) \\
& =\frac{1}{b} \int_{\|x\| \geq 1 /(\alpha C)}(\operatorname{Ei}(\alpha C\|x\|)-\operatorname{Ei}(1)) v_{L}(d x) .
\end{aligned}
$$

Since $v_{L}$ is a Lévy measure, $\int_{\|x\| \geq 1 /(\alpha C)} \operatorname{Ei}(1) v_{L}(d x)<\infty$ for all $\alpha>0$ and the integral $\int_{\|x\| \geq 1 /(\alpha C)} \operatorname{Ei}(\alpha C\|x\|) v_{L}(d x)$ is finite if and only if $\int_{\|x\| \geq 1} \operatorname{Ei}(\alpha C\|x\|) v_{L}(d x)<\infty$. Therefore (2.5) is sufficient for $\mathbb{E}\left(e^{\alpha\left\|Y_{1}\right\|}\right)<\infty$.

From (2.4) it follows that for any $c>0$ there exists a $K(c)>0$ such that $|\operatorname{Ei}(x)| \leq K(c) e^{x}$ for all $x \geq c$. This shows that (2.6) implies (2.5).

If $(q, p)=(1,0), A_{1}$ is diagonal or unitarily diagonalisable, $\|\cdot\|$ is the Euclidean norm and $B_{0}=I_{d}$, then one can take $b=-\max (\Re(\sigma(A)))$ and $C=1$. So a $d$-dimensional CARMA $(1,0)$ process (OU process) with unitarily diagonalisable $A$ has at least as many exponential moments as the driving Lévy process.

\subsection{Multivariate EGARCH processes in discrete time}

Multivariate EGARCH processes have been introduced recently in [16] as a natural extension of the univariate model of [22]. Yet, it should be noted that the definition below is more general than the one of [16]. For the necessary background on multivariate ARMA processes we refer to [5]. 
Definition 2.2 (Multivariate Discrete Time $\operatorname{EGARCH}(\mathrm{p}, \mathrm{q})$ ). Let $d, p, q \in \mathbb{N}, \mu \in \mathrm{S}_{d}, \alpha_{1}, \ldots$, $\alpha_{q}, \beta_{1}, \ldots, \beta_{p} \in M_{m}(\mathbb{R})$ with $m=\frac{d(d+1)}{2}, \epsilon=\left(\epsilon_{n}\right)_{n \in \mathbb{Z}}$ an i.i.d. sequence of $\mathbb{R}^{d}$-valued random variables with $\mathbb{E}\left(\epsilon_{1}\right)=0$ and $\operatorname{Var}\left(\epsilon_{1}\right)=I_{d}$ and $f: \mathbb{R}^{d} \rightarrow \mathbb{R}^{m}$ a measurable function such that $f\left(\epsilon_{1}\right) \in L^{2}$. Suppose $\alpha_{q} \neq 0, \beta_{p} \neq 0$ and that

$$
\operatorname{det}\left(1-\alpha_{1} z-\cdots-\alpha_{q} z^{q}\right) \neq 0
$$

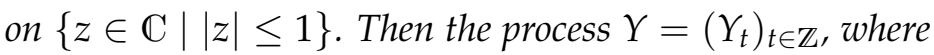

$$
Y_{t}=\exp \left(\left(\mu+H_{t}\right) / 2\right) \epsilon_{t}
$$

and the vectorised log volatility $H$ is given by

$$
\operatorname{vech}\left(H_{t}\right)=\sum_{k=1}^{p} \beta_{k} f\left(\epsilon_{t-k}\right)+\sum_{k=1}^{q} \alpha_{k} \operatorname{vech}\left(H_{t-k}\right)
$$

for all $t \in \mathbb{Z}$, is called an $\operatorname{EGARCH}(p, q)$ process.

Above we have considered a general transformation $f$ of the noise sequence $\epsilon$. Concrete specifications should be made in such a way that the model exhibits some desired properties, e.g. a leverage effect (i.e. an asymmetric response to positive and negative shocks). In the univariate case the "standard choice" introduced originally in [22] is

$$
f(\eta)=\theta \eta+\gamma\left(|\eta|-\mathbb{E}\left(\left|\epsilon_{1}\right|\right)\right)
$$

with some real parameters $\theta, \gamma$. This choice allows for a leverage effect, is at the same time of a simple structure and ensures $\mathbb{E}\left(f\left(\epsilon_{1}\right)\right)=0$. The logarithmic volatility models put forth in [16] can all be transformed into our above model using appropriate choices of $f$. However, all of them lead to functional forms involving only the individual components $\epsilon_{i, t}, i=1, \ldots, d$, of the innovation sequence $\epsilon$ and their absolute values $\left|\epsilon_{i, t}\right|$ in a linear manner. In particular, crossproducts of the form $\epsilon_{i, t} \epsilon_{j, t}$ do not enter the specification of $f$. Dependence on these crossproducts seems, however, desirable, especially when comparing things to multivariate GARCH specifications. We thus suggest two new possible choices for $f$ now. The first possible choice

$$
f(\eta)=\Theta \eta+\Gamma\left(\operatorname{vech}\left(\left(\eta \eta^{*}\right)^{1 / 2}\right)-\mathbb{E}\left(\operatorname{vech}\left(\left(\epsilon_{1} \epsilon_{1}^{*}\right)^{1 / 2}\right)\right)\right)
$$

with $\eta \in \mathbb{R}^{d}, \Theta \in M_{m, d}(\mathbb{R})$ and $\Gamma \in M_{m}(\mathbb{R})$ is a straightforward multivariate extension of the standard choice. Note that $\left(\eta \eta^{*}\right)^{1 / 2}$ can be interpreted as an extension of the absolute value to a multidimensional setting and that $\left(\left(\eta \eta^{*}\right)^{1 / 2}\right)_{i j}=\eta_{i} \eta_{j} /\|\eta\|_{2}$ with $\|\cdot\|_{2}$ denoting the Euclidean norm on $\mathbb{R}^{d}$. The second possibility we suggest is to use a generalised standard choice component-wise, viz.

$$
\begin{aligned}
f(\eta) & =\operatorname{vech}\left(g(\eta)-\mathbb{E}\left(g\left(\epsilon_{1}\right)\right)\right) \text { with } \\
g & : \mathbb{R}^{d} \rightarrow S_{d},\left(\eta_{1}, \eta_{2}, \cdots, \eta_{d}\right) \mapsto\left(g_{i j}\left(\eta_{i}, \eta_{j}\right)\right)_{1 \leq i, j \leq d} \\
g_{i i}\left(\eta_{i}, \eta_{i}\right) & :=\theta_{i, i} \eta_{i}+\gamma_{i, i}\left|\eta_{i}\right| \text { for } i=1,2, \ldots, d \\
g_{i j}\left(\eta_{i}, \eta_{j}\right) & :=\theta_{i, j} \frac{\eta_{i} \eta_{j}}{\sqrt{\left|\eta_{i} \eta_{j}\right|}}+\gamma_{i, j} \sqrt{\left|\eta_{i} \eta_{j}\right|} \text { for } i=1,2, \ldots, d, j=1,2, \ldots, i-1 \\
g_{i j}\left(\eta_{i}, \eta_{i}\right) & :=g_{j i}\left(\eta_{i}, \eta_{j}\right) \text { for } i=1,2, \ldots, d, j=i+1, i+2, \ldots, d
\end{aligned}
$$

where $\theta_{i, j}, \gamma_{i, j}$ with $i=1,2, \ldots, d, j=1,2 \ldots, i$ are real parameters.

The following proposition shows that $f$ as specified in (2.7) or (2.8) satisfies the required conditions for EGARCH processes. 
Proposition 2.3. Let $\epsilon_{1}$ be an $\mathbb{R}^{d}$-valued random variable with $\epsilon_{1} \in L^{2}$ and $f: \mathbb{R}^{d} \rightarrow \mathbb{R}^{m}$ as specified in Equation (2.7) or (2.8). Then $f$ is well-defined and $f\left(\epsilon_{1}\right) \in L^{2}$.

Proof. If $f$ is specified by (2.8) this follows from an element-wise application of the CauchySchwarz inequality.

If $f$ is given by (2.7) we are free to choose any norm for the proof. Thus we work in the following with the Euclidean norm $\|\cdot\|_{2}$ on $\mathbb{R}^{d}$, resp. $\mathbb{R}^{m}$, and the induced operator norm on matrix spaces. Elementary calculations give $\left\|\left(\epsilon_{1} \epsilon_{1}{ }^{*}\right)^{1 / 2}\right\|_{2}=\left\|\epsilon_{1}\right\|_{2}$, which implies the welldefinedness. Likewise, we use the operator norm $\|\cdot\|$ induced by these choices for the vech operator. We have

$$
\left\|f\left(\epsilon_{1}\right)\right\|_{2} \leq\|\Theta\|_{2}\left\|\epsilon_{1}\right\|_{2}+\|\Gamma\|_{2}\left(\|\operatorname{vech}\|\left\|\left(\epsilon_{1} \epsilon_{1}^{*}\right)^{1 / 2}\right\|_{2}+\left\|\mathbb{E}\left(\operatorname{vech}\left(\left(\epsilon_{1} \epsilon_{1}^{*}\right)^{1 / 2}\right)\right)\right\|_{2}\right) .
$$

Using Jensen's inequality one obtains

$$
\left\|\mathbb{E}\left(\operatorname{vech}\left(\left(\epsilon_{1} \epsilon_{1}^{*}\right)^{1 / 2}\right)\right)\right\|_{2} \leq\|\operatorname{vech}\| \mathbb{E}\left(\left\|\epsilon_{1}\right\|_{2}\right) .
$$

Thus

$$
\left\|f\left(\epsilon_{1}\right)\right\|_{2} \leq\left(\|\Theta\|_{2}+\|\Gamma\|_{2} \| \text { vech } \|\right)\left\|\epsilon_{1}\right\|_{2}+\|\Gamma\|_{2} \| \text { vech } \| \mathbb{E}\left(\left\|\epsilon_{1}\right\|_{2}\right) .
$$

Since $\epsilon_{1} \in L^{2}$ this immediately implies $f\left(\epsilon_{1}\right) \in L^{2}$.

\section{MULTIVARIATE EXPONENTIAL COGARCH}

\subsection{Definition and stationarity}

Now we define the exponential continuous time $\operatorname{GARCH}(p, q)$ process by specifying the vechtransformed $\log$-volatility process as a $\operatorname{CARMA}(q, p-1)$ process.

Definition 3.1. Let $L=\left(L_{t}\right)_{t \geq 0}$ be a d-dimensional zero-mean Lévy process with Lévy measure $v_{L}$ such that $\int_{\|x\| \geq 1}\|x\|^{2} v_{L}(d x)<\infty$ and associated jump measure $N_{L}$. Furthermore, let $h: \mathbb{R}^{d} \rightarrow \mathbb{R}^{m}$ with $m=\frac{d(d+1)}{2}$ be a measurable function satisfying

$$
\int_{\mathbb{R}^{d}}\|h(x)\|^{2} v_{L}(d x)<\infty,
$$

$p, q \in \mathbb{N}$ with $q \geq p$ and $A_{1}, \ldots, A_{q}, B_{0}, \ldots, B_{p-1} \in M_{m}(\mathbb{R})$ with $A_{q} \in G L_{d}(\mathbb{R})$ and $B_{0} \neq 0$ such that all zeros of the determinant $\operatorname{det}(Q(z))$ of the autoregressive polynomial $Q(z):=z^{q}+A_{1} z^{q-1}+$ $A_{2} z^{q-2}+\ldots+A_{q}, z \in \mathbb{C}$, have strictly negative real part.

Then we define the d-dimensional exponential $\operatorname{COGARCH}(p, q)$ process $G$, abbreviated to ECO$\operatorname{GARCH}(p, q)$, as the stochastic process satisfying,

$$
d G_{t}:=\exp \left(\left(\mu+H_{t-}\right) / 2\right) d L_{t}, \quad t>0, \quad G_{0}=0,
$$

where $\mu \in \mathbb{S}_{d}$ and the log-volatility process $H=\left(H_{t}\right)_{t \geq 0}$ is a process in $\mathbb{S}_{d}$ with vectorial state space representation

$$
\begin{aligned}
\operatorname{vech}\left(H_{t}\right) & :=\left(I_{m}, 0, \ldots, 0\right) X_{t}, \quad t \geq 0 \\
d X_{t} & =A X_{t} d t+\tilde{B} d M_{t}, \quad t>0
\end{aligned}
$$

with the initial value $X_{0} \in \mathbb{R}_{q m}$ being independent of the driving Lévy process $L$ and

$$
M_{t}:=\int_{0}^{t} \int_{\mathbb{R}^{d} \backslash\{0\}} h(x) \tilde{N}_{L}(d s, d x), \quad t \geq 0,
$$


being a zero-mean Lévy process. The matrices $A \in M_{q m}(\mathbb{R})$ and $\tilde{B} \in M_{q m, m}(\mathbb{R})$ are defined by

$$
A=\left(\begin{array}{ccccc}
0 & I_{m} & 0 & \cdots & 0 \\
0 & 0 & I_{m} & \cdots & 0 \\
\vdots & \vdots & \vdots & \ddots & \vdots \\
0 & 0 & 0 & \cdots & I_{m} \\
-A_{q} & -A_{q-1} & -A_{q-2} & \cdots & -A_{1}
\end{array}\right), \quad \tilde{B}=\left(\begin{array}{c}
\tilde{B}_{1} \\
\tilde{B}_{2} \\
\vdots \\
\tilde{B}_{q-1} \\
\tilde{B}_{q}
\end{array}\right)
$$

with coefficients $\tilde{B}_{q-j}=-\sum_{i=1}^{q-j-1} A_{i} \tilde{B}_{q-j-i}+B_{p-1-j}$ for $j=0,1, \ldots, q-1$ (setting $B_{i}=0$ for $i<0$ ). If $p=q=1$, we have $A=-A_{1}$ and $\tilde{B}=B_{0}$.

In a financial context $G$ is understood to be the log price process of $d$ stocks with volatility (instantaneous variance) process $\exp (\mu+H)$. Moreover, the log returns over a time interval of length $r>0$ ending at time $t$, which are especially relevant in a financial context, are described by the increments of $G$

$$
G_{t}^{(r)}:=G_{t}-G_{t-r}=\int_{(t-r, t]} \exp \left(\left(\mu+H_{s-}\right) / 2\right) d L_{s}, \quad t \geq r>0 .
$$

Thus our continuous time model gives us the possibility to model ultra high frequency data, which consists of returns over varying time intervals. On the other hand an equidistant sequence of such non-overlapping returns of length $r$ is given by $\left(G_{n r}^{(r)}\right)_{n \in \mathbb{N}}$. Such a sequence then corresponds to a discrete time multivariate EGARCH process $Y$.

Remark 3.2. (a) The condition (3.9) ensures that the integral defining the Lévy process $M$ is indeed well-defined and that $M$ has a finite variance.

(b) After extending the Léry process $\left(M_{t}\right)_{t \in \mathbb{R}^{+}}$to one defined on the whole real line the unique stationary version of $H$ can be written as

$$
\operatorname{vech}\left(H_{t}\right)=\int_{-\infty}^{t}\left(I_{m}, 0, \ldots, 0\right) e^{A(t-s)} \tilde{B} d M_{s} .
$$

(c) If $q \geq p+1$ the log-volatility process is continuous and $(q-p-1)$ times differentiable, which follows from the state space representation of $\operatorname{vech}(H)$ (cf. [20, Proposition 3.32]). In particular, the volatility will only contain jumps for $p=q$.

So far we have considered a general transformation $h$ of the jumps of the driving Lévy process $L$. Concrete specifications should be made in such a way that the model exhibits similar properties, e.g. a leverage effect, as in the discrete time case. The choice

$$
h(\eta)=\Theta \eta+\Gamma \operatorname{vech}\left(\left(\eta \eta^{*}\right)^{1 / 2}\right),
$$

with $\Theta \in M_{m, d}(\mathbb{R})$ and $\Gamma \in M_{m}(\mathbb{R})$, being the continuous time analogue of (2.7) clearly is always a valid choice, as an inspection of the proof of Proposition 2.3 shows. Again it is noteworthy that this extends the standard choice from the univariate literature.

A choice analogous to $(2.8)$ is

$$
h(\eta)=\operatorname{vech}(g(\eta))
$$

with $g$ as in (2.8). That $\int_{\mathbb{R}^{d}}\|h(x)\|^{2} v_{L}(d x)$ is finite is elementary to see.

Both specifications (3.13) and (3.14) obviously allow for asymmetric responses to positive and negative shocks in the logarithmic (co)variance components. Concrete examples for the choice of $\Theta$ and $\Gamma$ in (3.13) are given in Section 4 . 
Proposition 3.3. Let $H$ and $G$ be as in Definition 3.1 with $h$ satisfying (3.9). If the eigenvalues of $A$, which are the same as the zeros of $\operatorname{det}(Q(z))$, all have negative real parts and $X_{0}$ has the same distribution as $\int_{0}^{\infty} e^{A u} \tilde{B} d M_{u}$, then $X, H$ and $\exp ((\mu+H) / 2)$ are strictly stationary. Further $\left(G_{n r}^{(r)}\right)_{n \in \mathbb{N}}$ is strictly stationary.

Proof. The result on $X$ and $H$ follows from [20, Theorem 3.12]. If $H$ is stationary the stationarity of $\left(G_{n r}^{(r)}\right)_{n \in \mathbb{N}}$ is obvious, since the increments of $L$ are stationary and independent by definition.

Remark 3.4. Necessary and sufficient condition for the existence of a unique stationary volatility process exist up to now only in the univariate case. Suppose that $d=1, q \geq 1,\left(B_{0}, \ldots, B_{q}\right) \neq \mathbf{0}$ and the Lévy process $M$ is not deterministic. Then equations (3.10) and (3.11) have a unique strictly stationary solution $H$ if and only if $\mathbb{E}\left(\log ^{+}\left(\left|M_{1}\right|\right)\right)<\infty$ and all singularities of the meromorphic function $z \mapsto Q(z) / P(z)$ on the imaginary axis are removable. This result follows from [6, Theorem 4.2]. Moreover, [20, Proposition 3.30] show that a multivariate CARMA process has finite second moments if and only if the driving Léry process has finite second moments, provided $\tilde{B}$ is injective. This shows that in the univariate case the conditions of Definition 3.1 are (up to adding common zeros in $Q$ and $P$ ) basically the necessary and sufficient conditions for the existence of the logarithmic volatility process $H$ in $L^{2}$. We conjecture that a comparable result is true in the multivariate case, but this first requires extending the results of [6] to the multivariate case which is intricate and hence beyond the scope of the present paper.

\subsection{Mixing and second order properties}

Mixing properties (see [10] for a comprehensive treatment) are useful for a number of applications. In particular for asymptotic statistics, since consistency results and central limit theorems exist for mixing processes. Thus we will derive mixing properties of the strictly stationary volatility process and the return process. First we recall the definition of strong mixing, which is also called $\alpha$-mixing for a process with continuous time parameter.

Definition 3.5 ([9]). For a process $Y=\left(Y_{s}\right)_{s \geq 0}$ define the $\sigma$-algebras $\mathcal{F}_{[0, u]}^{Y}:=\sigma\left(\left(Y_{s}\right)_{s \in[0, u]}\right)$ and $\mathcal{F}_{[u+t, \infty)}^{Y}:=\sigma\left(\left(Y_{s}\right)_{s \geq u+t}\right)$ for all $u \geq 0$. Then $Y$ is called strongly or $\alpha$-mixing, if

$$
\begin{aligned}
\alpha(t) & =\sup _{u \geq 0} \alpha\left(\mathcal{F}_{[0, u]}^{Y}, \mathcal{F}_{[u+t, \infty)}^{Y}\right) \\
& :=\sup _{u \geq 0} \sup \left\{|P(A \cap B)-P(A) P(B)|: A \in \mathcal{F}_{[0, u]}^{Y}, B \in \mathcal{F}_{[u+t, \infty)}^{Y}\right\} \rightarrow 0,
\end{aligned}
$$

as $t \rightarrow \infty$.

Above we denote by $\sigma(\cdot)$ the generated completed $\sigma$-algebra. The strong mixing property with exponential rate of the log-volatility, volatility and return process is the subject of the next theorem. Here strong mixing with exponential rate (exponential $\alpha$-mixing) means that $\alpha(t)$ decays to zero exponentially fast for $t \rightarrow \infty$.

Theorem 3.6. Let vech $(H)$ be defined by (3.10) and (3.11). Assume that the eigenvalues of $A$ all have negative real parts and $X_{0}$ has the same distribution as $\int_{0}^{\infty} e^{A u} \tilde{B} d M_{u}$, hence $H$ and $\exp (\mu+H)$ are strictly stationary.

(i) Then there exist constants $K>0$ and $a>0$ such that

$$
\alpha_{H}(t) \leq K \cdot e^{-a t} \text { and } \quad \alpha_{\exp (\mu+H)}(t) \leq K \cdot e^{-a t}, \quad \text { as } t \rightarrow \infty,
$$

where $\alpha_{H}(t)$ and $\alpha_{\exp (\mu+H)}(t)$ are the $\alpha$-mixing coefficients of the log-volatility and volatility process, respectively. 
(ii) Then for all $r>0$ the discrete time process $\left(G_{n r}^{(r)}\right)_{n \in \mathbb{N}}$, where $G_{n r}^{(r)}$ is defined in (3.12), is strongly mixing with exponential rate and ergodic.

Proof. (i) Since vech $(H)$ is a CARMA $(q, p-1)$ process the result follows from [20, Proposition 3.34] and the fact that $\alpha$-mixing is preserved under continuous transformations.

(ii) The proof works along the lines of the proof of [13, Theorem 3.1].

Corollary 3.7. Let $\left(t_{n}\right)_{n \in \mathbb{N}_{0}}$ be a strictly increasing sequence of observation time points with $\lim _{n \rightarrow \infty} t_{n}=\infty$ and $t_{n}=k_{n} c$ for all $n \in \mathbb{N}_{0}$, where $k_{n} \in \mathbb{N}_{0}$ and $c>0$. Then the discrete time process $\left(G_{t_{n}}^{\left(\Delta_{n}\right)}\right)_{n \in \mathbb{N}}$,

$$
G_{t_{n}}^{\left(\Delta_{n}\right)}:=G_{t_{n}}-G_{t_{n-1}}
$$

with $\Delta_{n}=t_{n}-t_{n-1}$, is strongly mixing with exponential rate.

Proof. Simply expand the grid of observation times to an equidistant one with step size $c$. Then clearly

$$
\mathcal{F}_{1,2, \ldots, l}^{G^{(\Delta \cdot)}} \subset \mathcal{F}_{1,2, \ldots, t_{l} / c}^{G^{(c)}} \text { and } \mathcal{F}_{k+l, k+l+1, \ldots}^{G^{(\Delta \cdot)}} \subset \mathcal{F}_{t_{k+l} / c, t_{k+l+1} / c, \ldots}^{G^{(c)}},
$$

where $\mathcal{F}_{1,2, \ldots, l}^{G^{(\Delta .)}}$ is the $\sigma$-algebra generated from the random vectors $G_{t_{1}}^{\left(\Delta_{1}\right)}, \ldots, G_{t_{l}}^{\left(\Delta_{l}\right)}$ and the other $\sigma$-algebras are defined analogously. An application of Theorem 3.6 then provides the result.

Now we derive the second order moment structure of the return process $\left(G_{t}^{(r)}\right)_{t \geq r}$ considering only the case of a strictly stationary volatility process.

Proposition 3.8. Let $L$ be a Lévy process with $\mathbb{E}\left(L_{1}\right)=0$ and $\mathbb{E}\left(\left\|L_{1}\right\|^{2}\right)<\infty$. Assume that the log-volatility process $H$ is strictly stationary and $\mathbb{E}\left(\left\|\exp \left(\left(\mu+H_{t}\right) / 2\right)\right\|\right)<\infty$. Then $\mathbb{E}\left(\left\|G_{t}\right\|^{2}\right)<\infty$ for all $t \geq 0$, and for every $t, h \geq r>0$ it holds that

$$
\begin{aligned}
\mathbb{E} G_{t}^{(r)} & =0 \\
\mathbb{E}\left(G_{t}^{(r)}\left(G_{t}^{(r)}\right)^{*}\right) & =\int_{0}^{r} \mathbb{E}\left(\exp \left(\left(\mu+H_{s-}\right) / 2\right) \mathbb{E}\left(L_{1} L_{1}^{*}\right) \exp \left(\left(\mu+H_{s-}\right) / 2\right)\right) d s \\
\operatorname{Cov}\left(G_{t}^{(r)}, G_{t+h}^{(r)}\right) & =0 .
\end{aligned}
$$

The results follow analogously to the univariate case in [13, Proposition 5.1]. Note that the second order moment structure of $\operatorname{vech}(H)$ is clear from [20], whereas for the volatility $\exp (\mu+H)$ and the "squared returns" $G_{t}^{(r)}\left(G_{t}^{(r)}\right)^{*}$ the formulae obtained in the univariate case are already not explicit. Thus we refrain from stating them in our multivariate setting.

Regarding the finiteness of "exponential moments" of $H$ needed above we have the following result.

Proposition 3.9. (i) Let $\|\cdot\|_{*}$ be an algebra norm on $\mathrm{S}_{d}$ and the ECOGARCH log-volatility process $H$ be strictly stationary. Then

$$
\mathbb{E}\left(e^{\alpha_{1} \alpha_{2}\left\|H_{1}\right\|_{*}}\right)<\infty \text { with } \alpha_{1}, \alpha_{2}>0
$$

implies

$$
\mathbb{E}\left(\left\|\exp \left(\alpha_{1}\left(\mu+H_{1}\right)\right)\right\|_{*}^{\alpha_{2}}\right)<\infty .
$$

(ii) Let moreover $C>0$ be such that

$$
\sup _{x \in \mathbb{R}^{m},\left\|\operatorname{vech}^{-1}(x)\right\|_{*}=1}\left\{\left\|\operatorname{vech}^{-1}\left(\left(I_{m}, 0 \ldots, 0\right) e^{A s} \tilde{B} x\right)\right\|_{*}\right\} \leq C e^{-b s}
$$


for all $s \geq 0$ and some $b>0$. Then (3.15) is in turn implied by

$$
\int_{x \in \mathbb{R}^{d},\left\|\operatorname{vech}^{-1}(h(x))\right\|_{*} \geq 1} \operatorname{Ei}\left(\alpha_{1} \alpha_{2} C\left\|\operatorname{vech}^{-1}(h(x))\right\|_{*}\right) v_{L}(d x)<\infty
$$

or

$$
\int_{x \in \mathbb{R}^{d},\left\|\operatorname{vech}^{-1}(h(x))\right\|_{*} \geq 1} \exp \left(\alpha_{1} \alpha_{2} C\left\|\operatorname{vech}^{-1}(h(x))\right\|_{*}\right) v_{L}(d x)<\infty
$$

Proof. (i) Since $\|\cdot\|_{*}$ is an algebra norm, $\left\|\exp \left(\alpha_{1}\left(\mu+H_{1}\right)\right)\right\|_{*}^{\alpha_{2}} \leq e^{\alpha_{1} \alpha_{2}\|\mu\|_{*}} e^{\alpha_{1} \alpha_{2}\left\|H_{1}\right\|_{*}}$. This immediately shows (i).

(ii) The second part follows from Proposition 2.1 using the norm $\|\cdot\|=\left\|\operatorname{vech}^{-1}(\cdot)\right\|_{*}$ on $\mathbb{R}^{m}$ and the definition of $M$ implying $v_{M}(d x)=v_{L}\left(h^{-1}(d x)\right)$, because vech $(H)$ is an $m$ dimensional stationary CARMA process.

\subsection{Approximate Calculation of the Leverage effect}

Intuitively it seems obvious that our model is capable of reproducing the leverage effect (for the first asset) when one specifies the function $h$ in such a way that $h(\epsilon)_{1}$ is larger when $\epsilon_{1}$ is negative (price of the first asset goes down) than when $\epsilon_{1}$ is negative. However, quantifying the leverage effect in our model is a very intricate issue. Therefore, we will below only give an approximate calculation in the general case. However, in Section 4 we will show the presence in concrete simulated examples and also one general class of models in dimension two where the presence of the leverage effect can be shown rigorously. Note that we quantify the leverage effect by looking at the covariance $\operatorname{Cov}\left(\Delta G_{t}, \operatorname{vecp}\left(\exp \left(\mu+H_{t}\right)\right)\right)$ of a jump in the price process and the volatility immediately after the jump. It is easy to see that this quantity equals $\operatorname{Cov}\left(\Delta G_{t}, \Delta\left(\operatorname{vecp}\left(\exp \left(\mu+H_{t}\right)\right)\right)\right)$ if $\mathbb{E}\left(\Delta L_{t}\right)=0$. To make everything welldefined all these expectations and covariances have to be understood as being conditional on $\left\|\Delta L_{t}\right\|>\epsilon$ for some $\epsilon>0$ (if $L$ is a compound Poisson process, $\epsilon=0$ may also be taken). Based on this quantity we say that the leverage effect is present (in e.g. the first component) if $\left(\operatorname{Cov}\left(\Delta G_{t}, \operatorname{vecp}\left(\exp \left(\mu+H_{t}\right)\right)\right)\right)_{11}<0$ (for all "sufficiently small" minimal jump sizes $\epsilon$ ).

One of the main problems, why it is much more complicated to quantify the leverage effect compared to the univariate case, is the following. In the univariate case the sign of $\Delta G_{t}$ equals the sign of $\Delta L_{t}$. However, in the multivariate case $(\Delta G)_{1, t}=\sum_{i=1}^{d}\left(\exp \left(\left(\mu+H_{t-}\right) / 2\right)\right)_{1 i}\left(\Delta L_{i, t}\right)$ maybe for instance negative and $\left(\Delta L_{1, t}\right)$ positive, since the current covariance structure allows also jumps in the other components of $L$ to affect $\left(\Delta G_{1, t}\right)$. Another problem is that the matrix exponential is not an operator monotone function (see [3, Problem V.5.1] or [14, p. 554]). This means that if $X, Y \in \mathbb{S}_{d}$ satisfy $X \geq Y$, i.e. $X-Y \in \mathbb{S}_{d}^{+}$, this does not imply that $e^{X} \geq e^{Y}$. Likewise no componentwise monotonicity holds, since in principle all components of $X \in S_{d}$ contribute to, say, $\left(e^{X}\right)_{11}$. These problems are probably also the reason why [16] claims but does not show that his models may capture the leverage effect.

Now we give an approximate calculation quantifying the leverage effect.

Denoting Frechet/total differentials with $D$ and setting $f: M_{d}(\mathbb{R}) \rightarrow M_{d}(\mathbb{R}), X \mapsto \exp (X)$ we have

$$
D f(A): M_{d}(\mathbb{R}) \rightarrow M_{d}(\mathbb{R}), X \mapsto \int_{0}^{1} e^{(1-t) A} X e^{t A} d t
$$

see [3, Example X.4.2 (v)]. Below all expectations and covariances have formally to be understood as being conditional on $\left\|\Delta L_{t}\right\|>\epsilon$ with some $\epsilon>0$. If $L$ is compound Poisson, we can take $\epsilon=0$. Let $G$ now be an $\operatorname{ECOGARCH}(p, p)$ process driven by a Lévy process $L$ satisfying 
$\mathbb{E}\left(\Delta L_{t}\right)=0$. Then using a first order Taylor approximation

$$
\begin{aligned}
& \operatorname{Cov}\left(\Delta G_{t}, \operatorname{vecp}\left(\exp \left(\mu+H_{t}\right)\right)^{*}\right) \\
& =\mathbb{E}\left(\exp \left(\left(\mu+H_{t-}\right) / 2\right) \Delta L_{t} \operatorname{vecp}\left(\exp \left(\mu+H_{t-}+\operatorname{vech}^{-1}\left(\tilde{B}_{1} \Delta M_{t}\right)\right)\right)^{*}\right) \\
& \approx \mathbb{E}\left(\exp \left(\left(\mu+H_{t-}\right) / 2\right) \Delta L_{t} \operatorname{vecp}\left(\exp \left(\mu+H_{t-}\right)\right)^{*}\right)+\mathbb{E}\left(\exp \left(\left(\mu+H_{t-}\right) / 2\right) \Delta L_{t}\right. \\
& \left.\quad \times \int_{0}^{1} \operatorname{vecp}\left(\exp \left((1-u)\left(\mu+H_{t-}\right)\right) \operatorname{vech}^{-1}\left(\tilde{B}_{1} \Delta M_{t}\right) \exp \left(u\left(\mu+H_{t-}\right)\right)\right)^{*} d u\right) \\
& =\mathbb{E}\left(\exp \left(\left(\mu+H_{t-}\right) / 2\right) \Delta L_{t} \operatorname{vecp}\left(\operatorname{vech}^{-1}\left(\tilde{B}_{1} \Delta M_{t}\right)\right)^{*}\right. \\
& \left.\quad \times \int_{0}^{1} \exp \left(u\left(\mu+H_{t-}\right)\right) \otimes \exp \left((1-u)\left(\mu+H_{t-}\right)\right), d u\right)
\end{aligned}
$$

Hence, using the stochastic continuity of $H$

$$
\begin{aligned}
& \operatorname{vecp}\left(\operatorname{Cov}\left(\Delta G_{t}, \operatorname{vecp}\left(\exp \left(\mu+H_{t}\right)\right)^{*}\right)\right) \\
& \approx \mathbb{E}\left(\int_{0}^{1} \exp \left(u\left(\mu+H_{t-}\right)\right) \otimes \exp \left((1-u)\left(\mu+H_{t-}\right)\right) d u \otimes \exp \left(\left(\mu+H_{t-}\right) / 2\right)\right) \\
& \quad \times \mathbb{E}\left(\operatorname{vecp}\left(\Delta L_{t} \operatorname{vecp}\left(\operatorname{vech}^{-1}\left(\tilde{B}_{1} \Delta M_{t}\right)\right)^{*}\right)\right) \\
& =\mathbb{E}\left(\int_{0}^{1} \exp \left(u\left(\mu+H_{t}\right)\right) \otimes \exp \left((1-u)\left(\mu+H_{t}\right)\right) d u \otimes \exp \left(\left(\mu+H_{t}\right) / 2\right)\right) \\
& \quad \times \mathbb{E}\left(\operatorname{vecp}\left(\operatorname{vech}^{-1}\left(B_{0} h\left(\Delta L_{t}\right)\right) \otimes \Delta L_{t}\right)\right) .
\end{aligned}
$$

A very nice property of the above expression is that this approximation of $\operatorname{Cov}\left(\Delta G_{t}\right.$, $\left.\operatorname{vecp}\left(\exp \left(\mu+H_{t}\right)\right)^{*}\right)$ factorises into one quantity which only depends on the stationary distribution of $H$ and a second factor depending only on the jumps of $L$. The second factor can be easily calculated from the Lévy measure of $L$ as

$$
\mathbb{E}\left(\operatorname{vecp}\left(\operatorname{vech}^{-1}\left(B_{0} h\left(\Delta L_{t}\right)\right) \otimes \Delta L_{t}\right)\right)=v_{L}(\|x\|>\epsilon)^{-1} \int_{\|x\|>\epsilon} \operatorname{vecp}\left(\operatorname{vech}^{-1}\left(B_{0} h(x)\right) \otimes x\right) v_{L}(d x)
$$

and regarding the first factor one should note that the stationary distribution of $H$ is known via its characteristic function/characteristic triplet (see [20, Proposition 3.27]), since $H$ is an MCARMA process. The second factor also resembles our intuition that we have the leverage effect, if $B_{0}$ and $h$ are such that $B_{0} h(x)$ is bigger for "negative" $x$ than for "positive" ones. Of course, this is only valid when the first factor is such that the signs of the elements corresponding to the variance (=diagonal) components of $\exp \left(\mu+H_{t}\right)$ are preserved.

Let us illustrate this with a concrete example where we without loss of generality consider the first component. Assume $h$ is of the form (3.13) and the components of $L$ are completely independent, i.e. if $L$ jumps then only one component jumps or in other words $v_{L}$ is concentrated on the axes. Then we have that

$$
\begin{aligned}
\mathbb{E}\left(\operatorname{vecp}\left(\operatorname{vech}^{-1}\left(B_{0} h\left(\Delta L_{t}\right)\right) \otimes \Delta L_{t}\right)_{1}\right) & =\mathbb{E}\left(\left(B_{0} h\left(\Delta L_{t}\right)\right)_{11} \Delta L_{1, t}\right) \\
& =\mathbb{E}\left(\left(\sum_{i=1}^{m} B_{0,1 i}\left(\Theta_{i 1} \Delta L_{1, t}+\Gamma_{i 1}\left|\Delta L_{1, t}\right|\right)\right) \Delta L_{1, t}\right)
\end{aligned}
$$


This shows - assuming the first factor in (3.17) does not change the sign of the first component - that we have a leverage effect in the first component when $\sum_{i=1}^{m} B_{0,1 i}\left(\Theta_{i 1} \Delta L_{1, t}+\Gamma_{i 1}\left|\Delta L_{1, t}\right|\right)$ is always positive, but larger for negative values of $\Delta L_{t}$ than for positive ones, and the jumps of $L$ have a symmetric distribution. Thus, like in the standard univariate case we have the leverage effect in the first component if $\Delta L$ has a symmetric distribution and $B_{0,1 i}, \Gamma_{i, 1},-\Theta_{i 1}, \Gamma_{i, 1}+\Theta_{i, 1} \geq$ 0 for all $i=1, \ldots, m$.

\subsection{Approximation of ECOGARCH(1,1) processes by EGARCH(1,1) processes}

In this section we summarise a result of [29] which provides an important link to discrete time EGARCH models and may serve as a starting point for estimating ECOGARCH(1,1) processes based on discrete observations. As [29] is concerned with approximations of SDEs in general and the presentation and lengthy proofs there are rather technical, it seems worthwhile to summarise the results for the ECOGARCH $(1,1)$ process here.

For the rest of the section we will just consider the ECOGARCH(1,1) process $G$ satisfying,

$$
d G_{t}=\exp \left(\left(\mu+H_{t-}\right) / 2\right) d L_{t}, \quad t>0, \quad G_{0}=0,
$$

where the vectorised $\log$-volatility process $X_{t}:=\left(\operatorname{vech}\left(H_{t}\right)\right)_{t \geq 0}$ is the process in $\mathbb{R}^{m}$ satisfying

$$
d X_{t}=-A_{1} X_{t} d t+B_{0} d M_{t}, \quad t>0,
$$

with the initial value $X_{0} \in \mathbb{R}^{m}$ being independent of the driving Lévy process $L$. In [29] a first jump approximation of multivariate Lévy driven stochastic differential equations is introduced. This result was then used to show the convergence of a sequence of piecewise constant processes determined by discrete time $\operatorname{EGARCH}(1,1)$ to the $\operatorname{ECOGARCH}(1,1)$ process in the Skorokhod topology in probability. For a complete and separable normed space $\left(E,\|\cdot\|_{E}\right)$ we denote the convergence of a sequence $\left(Z^{(n)}\right)_{n \in \mathbb{N}}$ of $E$-valued càdlàg random processes in probability in the Skorokhod topology to a càdlàg random process $Z$ by $\operatorname{plim}_{n \rightarrow \infty} d_{E}\left(Z^{(n)}, Z\right)=0$ with plim denoting the limit in probability and $d_{E}$ is a metric inducing the Skorokhod topo$\operatorname{logy}$ (see e.g. [18]). The result is then the following

Theorem 3.10 ([29], Theorem 4.1). Let $(G, X)$ in $\mathbb{R}^{d} \times \mathbb{R}^{m}$ be a d-dimensional ECOGARCH $(1,1)$ process $G$ and its associated vectorised log-volatility process $X=\operatorname{vech}(H)$ with initial value $\left(G_{0}, X_{0}\right)$. Let $\left(t_{i}^{(n)}\right)_{i \in \mathbb{N}_{0}}$ for each $n \in \mathbb{N}$ be a strictly increasing sequence in $\mathbb{R}^{+}$with $t_{0}^{(n)}=0$ and $\lim _{i \rightarrow \infty} t_{i}^{(n)}=$ $\infty$. Defining $\delta^{(n)}=\sup _{i \in \mathbb{N}}\left\{t_{i}^{(n)}-t_{i-1}^{(n)}\right\}$ assume that $\lim _{n \rightarrow \infty} \delta^{(n)}=0$.

Then there exists for each $n \in \mathbb{N}$ a function $h_{n}: \mathbb{R}^{d} \times \mathbb{R}^{+} \rightarrow \mathbb{R}^{m}$ and a sequence of independent random variables $\left(\epsilon_{i}^{(n)}\right)_{i \in \mathbb{N}}$ in $\mathbb{R}^{d}$ with finite variance and $\mathbb{E}\left(\epsilon_{i}^{(n)}\right)=0 \forall i, n \in \mathbb{N}$ such that $h_{n}\left(\epsilon_{i}^{(n)}, t_{i}^{(n)}-t_{i-1}^{(n)}\right)$ has finite variance, $\mathbb{E}\left(h_{n}\left(\epsilon_{i}^{(n)}, t_{i}^{(n)}-t_{i-1}^{(n)}\right)\right)=0$ and

$$
\operatorname{plim}_{n \rightarrow \infty} d_{\mathbb{R}^{d} \times \mathbb{R}^{m}}\left(\left(I Y^{(n)}, X^{(n)}\right),(G, X)\right)=0,
$$

where for each $n \in \mathbb{N}$ the process $\left(I Y^{(n)}, X^{(n)}\right)$ in $\mathbb{R}^{d} \times \mathbb{R}^{m}$ is defined by

$$
\begin{aligned}
\left(I Y_{0}^{(n)}, X_{0}^{(n)}\right) & =\left(G_{0}, X_{0}\right), \\
I Y_{t_{i}^{(n)}}^{(n)} & =I Y_{t_{i-1}^{(n)}}^{(n)}+\exp \left(\left(\mu+\operatorname{vech}^{-1}\left(X_{t_{i-1}^{(n)}}^{(n)}\right)\right) / 2\right) \epsilon_{i}^{(n)}, \\
X_{t_{i}^{(n)}}^{(n)} & =e^{-A_{1}\left(t_{i}^{(n)}-t_{i-1}^{(n)}\right)} X_{t_{i-1}^{(n)}}^{(n)}+B_{0} h_{n}\left(\epsilon_{i}^{(n)}, t_{i}^{(n)}-t_{i-1}^{(n)}\right) \text { for all } i \in \mathbb{N} \text { and } \\
\left(I Y_{t}^{(n)}, X_{t}^{(n)}\right) & =\left(I Y_{t_{i-1}^{(n)}}^{(n)}, X_{t_{i-1}^{(n)}}^{(n)}\right) \text { for } t \in\left(t_{i-1}^{(n)}, t_{i}^{(n)}\right), i \in \mathbb{N} .
\end{aligned}
$$


The sequence $\left(\epsilon_{i}^{(n)}\right)_{i \in \mathbb{N}}$ can be chosen to be i.i.d. provided $t_{i}^{(n)}-t_{i-1}^{(n)}=\delta^{(n)}$ for all $i \in \mathbb{N}$.

If $h$ is continuous, $h_{n}$ can be chosen such that the sequence of functions $h_{n}: \mathbb{R}^{d} \times \mathbb{R}^{+} \rightarrow \mathbb{R}^{m}$ satisfies

$$
\lim _{n \rightarrow \infty}\left(\sup _{z \in K} \sup _{i \in \mathbb{N}}\left\{\left\|h_{n}\left(z, t_{i}^{(n)}-t_{i-1}^{(n)}\right)-h(z)\right\|\right\}\right)=0
$$

for all compact $K \subset \mathbb{R}^{d}$. If $h$ is uniformly continuous, $h_{n}$ can be chosen such that (3.18) holds with $\mathbb{R}^{d}$ instead of $K$.

When the time grids are equidistant, i.e. $t_{i}^{(n)}-t_{i-1}^{(n)}=\delta^{(n)}$ for all $i \in \mathbb{N}$, and $\left(\epsilon_{i}^{(n)}\right)_{i \in \mathbb{N}}$ is chosen i.i.d., then the increments $\left(Y_{t_{i}^{(n)}}^{(n)}\right)_{i \in \mathbb{N}}:=\left(I Y_{t_{i}^{(n)}}^{(n)}-I Y_{t_{i-1}^{(n)}}^{(n)}\right)_{i \in \mathbb{N}}$ of $I Y^{(n)}$ are a discrete time multivariate $\operatorname{EGARCH}(1,1)$ process with associated vectorised log-volatility process $\left(X_{t_{i-1}^{(n)}}^{(n)}\right)_{i \in \mathbb{N}}$. Only $\operatorname{Var}\left(\epsilon_{i}^{(n)}\right)=I_{d}$ will usually not be satisfied, but [29, Theorem 4.4] provides a variant of the above statement ensuring also this property up to a scaling corresponding to the size of the time grid.

Remark 3.11. The function $h_{n}$ in Theorem 3.10 can be specified as $h_{n}: \mathbb{R}^{d} \times \mathbb{R}^{+} \rightarrow \mathbb{R}^{m}$,

$$
(z, t) \mapsto h\left(z+\frac{1-e^{-v_{L}\left(J^{(n)}\right) t}}{v_{L}\left(J^{(n)}\right)} \int_{J^{(n)}} x v_{L}(d x)\right)-\frac{1-e^{-v_{L}\left(J^{(n)}\right) t}}{v_{L}\left(J^{(n)}\right)} \int_{J^{(n)}} h(x) v_{L}(d x),
$$

where $J^{(n)}=\left\{x \in \mathbb{R}^{d}:\left\|\left(x^{*}, h(x)^{*}\right)^{*}\right\|>m^{(n)}\right\}$ and $\left(m^{(n)}\right)_{n \in \mathbb{N}}$ is a positive sequence such that condition (3.1) in [29] is satisfied. Based on a choice for $h$ and given observations $G_{t_{1}}, \ldots, G_{t_{n}}$ a quasi maximum likehood type estimator for the unknown parameters could be defined similar as in [19].

\section{EXAMPLES AND SIMULATIONS}

In this section we demonstrate how to choose the parameters in the model to obtain a leverage effect. We simulate sample path trajectories for three different examples. The first two examples are such that a leverage effect is present. To empirically control the leverage effect we compute estimates for the following quantities

$$
\operatorname{Corr}\left(\Delta G_{1, t^{1, *},} \exp \left(\mu+H_{t^{1, *}}\right)_{11}\right) \quad \text { and } \quad \operatorname{Corr}\left(\Delta G_{2, t^{2, *},} \exp \left(\mu+H_{t^{2, *}}\right)_{22}\right),
$$

where $t^{i, *}, i=1,2$, is a jump time in the $i$-th component.

As a first example we consider a bivariate ECOGARCH $(1,1)$ process. The driving Lévy process $L$ has the characteristic function

$$
\mathbb{E}\left[e^{i\left\langle u, L_{t}\right\rangle}\right]=\exp \left[-\frac{1}{2}\left\langle u, I_{2} u\right\rangle+\int_{\mathbb{R}^{2}}\left(e^{i\langle u, x\rangle}-1\right) v_{L}(d x)\right],
$$

where $v_{L}$ is a finite measure with density

$$
f(x)=\lambda \sqrt{\frac{n_{1} \lambda}{n_{1}-2}} \sqrt{\frac{n_{2} \lambda}{n_{2}-2}} t_{n_{1}}\left(\sqrt{\frac{n_{1} \lambda}{n_{1}-2}} x_{1}\right) t_{n_{2}}\left(\sqrt{\frac{n_{2} \lambda}{n_{2}-2}} x_{2}\right)
$$

and $t_{n}$ denotes the density of the $t$-distribution with $n$ degrees of freedom. In this particular example we choose $n_{1}=4, n_{2}=10$ and the rate $\lambda$ is set equal to 2 . The log-volatility process $H$ has the vectorial state space representation 


$$
\begin{aligned}
\operatorname{vech}\left(H_{t}\right) & =X_{t} \\
d X_{t} & =-A_{1} X_{t} d t+\tilde{B}_{1} d M_{t}
\end{aligned}
$$

with

$$
-A_{1}=\left(\begin{array}{rrr}
-1.0490 & -1.5078 & -0.4814 \\
-0.1496 & 0.1065 & 0.5105 \\
1.1074 & 0.6021 & -0.9310
\end{array}\right), \tilde{B}_{1}=B_{0}=I_{3}
$$

The Lévy process $M$ is defined by the function

$$
h(\eta)=\left(\begin{array}{ll}
-0.40 & -0.40 \\
-0.01 & -0.01 \\
-0.40 & -0.40
\end{array}\right) \eta+\left(\begin{array}{ccc}
0.01 & 0 & 0 \\
0 & 0.1 & 0 \\
0 & 0 & 0.01
\end{array}\right) \operatorname{vech}\left(\left(\eta \eta^{*}\right)^{1 / 2}\right) .
$$

From the choice of $\Theta$ and $\Gamma$ it follows that future volatility should be negatively correlated with current jumps in the price. The remaining parameter $\mu \in \mathrm{S}_{2}$ is set to $\left(\begin{array}{cc}-8 & 0 \\ 0 & -8\end{array}\right)$.

In Figure 1 parts of the trajectories of the bivariate log-price $G$ as well as the diagonal elements of the volatility process $\exp (\mu+H)$ are shown. The trajectories of the log-volatility process where simulated by applying a stochastic Euler scheme over the time points consisting of the jump times of the two compound Poisson processes and a grid with step size 0.01.
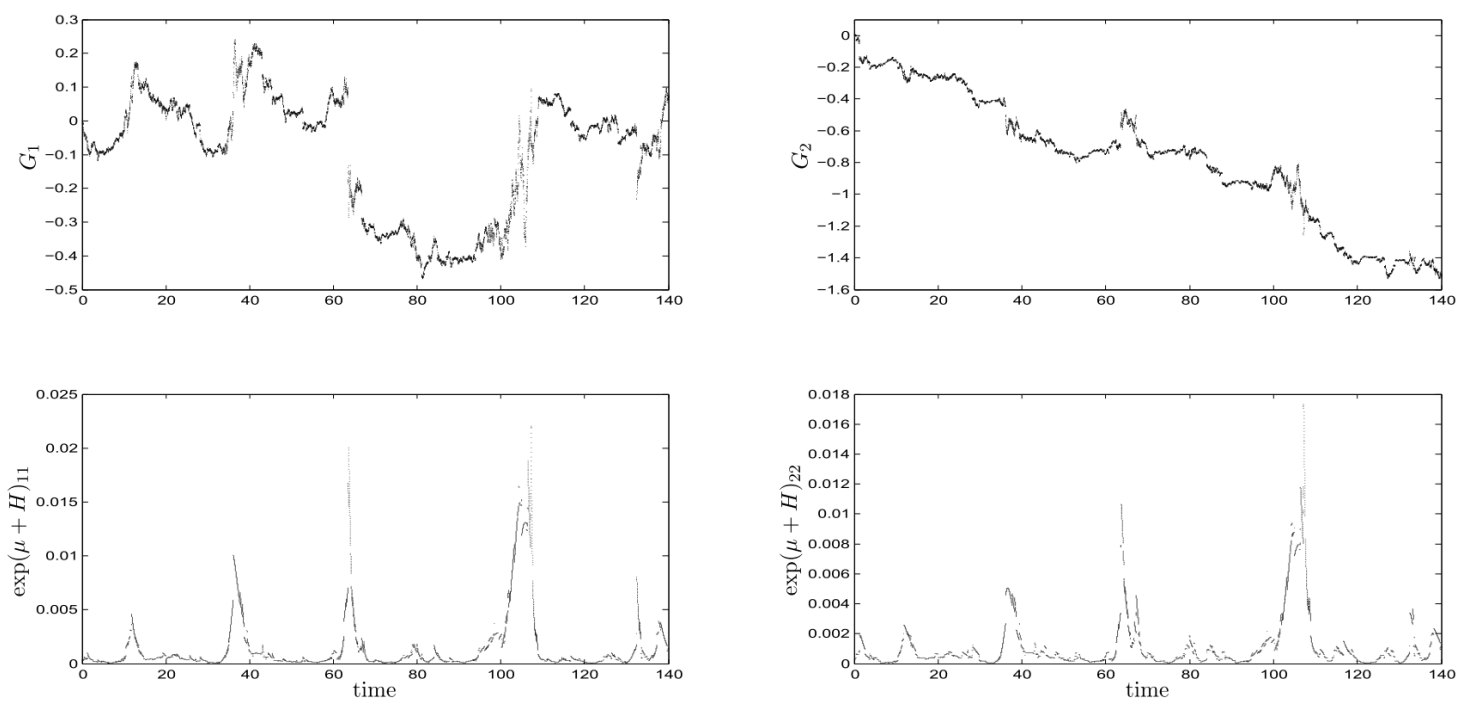

Figure 1: Simulated trajectories of the log-price process $G$ in the top row and the diagonal elements of the volatility process $\exp (\mu+H)$ in the bottom row.

The driving Lévy process $L$ has independent components. Nevertheless we get dependent volatilities $\exp (\mu+H)_{11}$ and $\exp (\mu+H)_{22}$ due to the choice of parameters, as can be seen from the empirical estimate of the crosscorrelation function $\rho_{12}(h)=\operatorname{Corr}\left(\exp \left(\mu+H_{t+h}\right)_{11}, \exp (\mu+\right.$ $\left.H_{t}\right)_{22}$ ) in Figure 2, where a lag of one corresponds to 0.01 units of time.

To estimate the quantities in (4.19) we simulated the trajectories 1000 times and then averaged over the 1000 estimates to get

$$
\begin{aligned}
& \widehat{\operatorname{Corr}}\left(\Delta G_{1, t^{1, *}, \exp }\left(\mu+H_{t^{1, *}}\right)_{11}\right)=-0.4665 \\
& \widehat{\operatorname{Corr}}\left(\Delta G_{2, t^{2, *},} \exp \left(\mu+H_{t^{2, *}}\right)_{22}\right)=-0.4570 \text {. }
\end{aligned}
$$



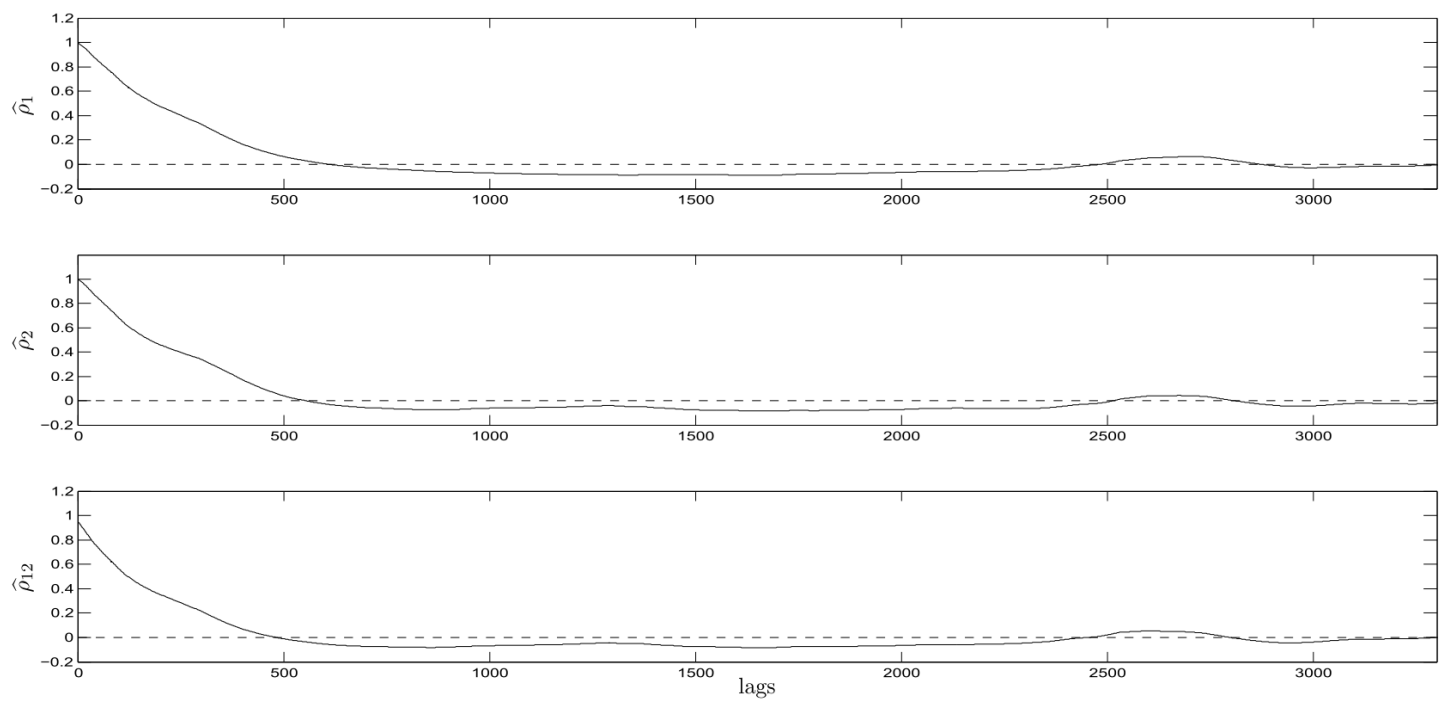

Figure 2: Empirical autocorrelation function $\widehat{\rho}_{1}$ (top) and $\widehat{\rho}_{2}$ (middle) of $\exp (\mu+H)_{11}$ and $\exp (\mu+H)_{22}$, respectively, and the empirical crosscorrelationfunction $\widehat{\rho}_{12}$ (bottom).

The corresponding empirical standard errors are 0.0083 and 0.0074 , respectively.

This empirical result is also confirmed by the following Proposition.

Proposition 4.1. Let $d=2$ and $\mathrm{G}$ a d-dimensional ECOGARCH $(p, p)$ process with $h$ given by (3.13). Assume that the driving Lévy process $L$ has independent components and that the distribution of the jumps of $L_{k}, k=1,2$, is symmetric, i.e. for all $\epsilon>0$,

$$
P\left(\Delta L_{k, t} \in d x|| \Delta L_{k, t} \mid>\epsilon\right)=P\left(\Delta L_{k, t} \in-d x|| \Delta L_{k, t} \mid>\epsilon\right), \quad t \geq 0, k=1,2 .
$$

Then conditionally on the event $\left|\Delta L_{k, t}\right|>\epsilon$, the sign of

$$
\operatorname{Cov}\left(\Delta G_{k, t}, \exp \left(\mu+H_{t}\right)_{k k}|| \Delta L_{k, t} \mid>\epsilon\right)
$$

is negative if

$$
\left\{\begin{array}{l}
\left(\tilde{B}_{1} \Theta\right)_{11}=\left(\tilde{B}_{1} \Theta\right)_{31}<0 \quad \text { and } \quad\left(\tilde{B}_{1} \Theta\right)_{21} \leq 0, \quad k=1 \\
\left(\tilde{B}_{1} \Theta\right)_{32}=\left(\tilde{B}_{1} \Theta\right)_{12}<0 \quad \text { and } \quad\left(\tilde{B}_{1} \Theta\right)_{22} \leq 0, \quad k=2
\end{array} .\right.
$$

Proof. In case $\left|\Delta L_{k, t}\right|>\epsilon$ and $\Delta L_{i, t}=0$ for some timepoint $t$, the log-volatility matrix is equal to

$$
H_{t}=\left(\begin{array}{ll}
h_{1, t} & h_{2, t} \\
h_{2, t} & h_{3, t}
\end{array}\right)
$$

where

$$
h_{j, t}=X_{j, t-}+\left(\tilde{B}_{1} \Theta\right)_{j k} \Delta L_{k, t}+\left(\tilde{B}_{1} \Gamma\right)_{j i(k)}\left|\Delta L_{k, t}\right|, \quad j=1,2,3,
$$

and $i(k)=\mathbb{1}_{\{1\}}(k)+3 \mathbb{1}_{\{2\}}(k)$.

The volatility matrix at time $t$ is then given by

$$
\exp \left(\mu+H_{t}\right)=\frac{1}{\tau}\left(\begin{array}{ll}
\sigma_{11, t}^{2} & \sigma_{12, t}^{2} \\
\sigma_{12, t}^{2} & \sigma_{22, t}^{2}
\end{array}\right)
$$

with

$$
\begin{aligned}
& \sigma_{11, t}^{2}=e^{\left(\mu_{11}+\mu_{22}+h_{1}+h_{3}\right) / 2}\left(\tau \cosh \left(\frac{\tau}{2}\right)+\left(\mu_{11}-\mu_{22}+h_{1}-h_{3}\right) \sinh \left(\frac{\tau}{2}\right)\right) \\
& \sigma_{12, t}^{2}=2\left(\mu_{12}+h_{2}\right) e^{\left(\mu_{11}+\mu_{22}+h_{1}+h_{3}\right) / 2} \sinh \left(\frac{\tau}{2}\right) \\
& \sigma_{22, t}^{2}=e^{\left(\mu_{11}+\mu_{22}+h_{1}+h_{3}\right) / 2}\left(\tau \cosh \left(\frac{\tau}{2}\right)+\left(\mu_{22}-\mu_{11}+h_{3}-h_{1}\right) \sinh \left(\frac{\tau}{2}\right)\right)
\end{aligned}
$$


and $\tau=\sqrt{\left(\mu_{11}-\mu_{22}+h_{1}-h_{3}\right)^{2}+4\left(\mu_{12}+h_{2}\right)^{2}}$ (see e.g. [26]).

Since the distribution of the jumps of $L_{k}$ is symmetric, we obtain

$$
\mathbb{E}\left(\Delta G_{k, t}|| \Delta L_{k, t} \mid>\epsilon\right)=0 .
$$

Define $P_{\epsilon}^{L}(d x)=P\left(\Delta L_{1, t} \in d x|| \Delta L_{1, t} \mid>\epsilon\right)$. Then we get for $k=1$

$$
\begin{aligned}
\operatorname{Cov}\left(\Delta G_{1, t}, \exp \left(\mu+H_{t}\right)_{11}|| \Delta L_{1, t} \mid>\epsilon\right) & =\mathbb{E}\left(\Delta G_{1, t} \exp \left(\mu+H_{t}\right)_{11}|| \Delta L_{1, t} \mid>\epsilon\right) \\
& =\int_{\left\{X_{t-}^{-1}\left(\mathbb{R}^{3}\right)\right\}} \exp \left(\frac{1}{2}\left(\mu+H_{t}(\omega)\right)\right)_{11} I(\omega) d P(\omega),
\end{aligned}
$$

where

$$
\begin{aligned}
I(\omega)= & \int_{x>\epsilon} x e^{\frac{1}{2}\left(\mu_{11}+\mu_{22}+X_{1, t-}(\omega)+X_{3, t-}(\omega)+\left(\left(\tilde{B}_{1} \Gamma\right)_{11}+\left(\tilde{B}_{1} \Gamma\right)_{31}\right) x\right)} \\
& \left\{e^{\left.\left(\tilde{B}_{1} \Theta\right)_{11}+\left(\tilde{B}_{1} \Theta\right)_{31}\right) \frac{x}{2}}\left[\cosh \left(\frac{\tau^{+}(x)}{2}\right)+\frac{\tilde{\tau}^{+}(x)}{\tau^{+}(x)} \sinh \left(\frac{\tau^{+}(x)}{2}\right)\right]\right. \\
& \left.-e^{\left.-\left(\tilde{B}_{1} \Theta\right)_{11}+\left(\tilde{B}_{1} \Theta\right)_{31}\right) \frac{x}{2}}\left[\cosh \left(\frac{\tau^{-}(x)}{2}\right)+\frac{\tilde{\tau}^{-}(x)}{\tau^{-}(x)} \sinh \left(\frac{\tau^{-}(x)}{2}\right)\right]\right\} P_{\epsilon}^{L}(d x)
\end{aligned}
$$

with

$$
\begin{aligned}
& \tilde{\tau}^{s}(x)=\mu_{11}-\mu_{22}+h_{1, t}^{s}(x)-h_{3, t}^{s}(x), \\
& \tau^{s}(x)=\sqrt{\left(\tilde{\tau}^{s}(x)\right)^{2}+4\left(\mu_{12}+h_{2, t}^{s}(x)\right)^{2}}
\end{aligned}
$$

and

$$
h_{j, t}^{s}(x)=\left\{\begin{array}{ll}
X_{j, t-}+\left(\tilde{B}_{1} \Theta\right)_{j 1} x+\left(\tilde{B}_{1} \Gamma\right)_{j 1} x, & s=+ \\
X_{j, t-}-\left(\tilde{B}_{1} \Theta\right)_{j 1} x+\left(\tilde{B}_{1} \Gamma\right)_{j 1} x, & s=-
\end{array}, \quad j=1,2,3 .\right.
$$

An inspection of the integrand of $I(\omega)$ reveals that $I(\omega)$ is almost surely negative if $\left(\tilde{B}_{1} \Theta\right)_{11}=\left(\tilde{B}_{1} \Theta\right)_{31}<0$ and $\left(\tilde{B}_{1} \Theta\right)_{21} \leq 0$, which implies that the sign of

$$
\operatorname{Cov}\left(\Delta G_{1, t}, \exp \left(\mu+H_{t}\right)_{11}|| \Delta L_{1, t} \mid>\epsilon\right)
$$

is negative. The same reasoning leads to the desired result for $k=2$.

Remark 4.2. Jumps in the $k$-th component of $G$ can of course also occur due to jumps in the j-th component in $L, j \neq k$. The sign of

$$
\operatorname{Cov}\left(\Delta G_{k, t}, \exp \left(\mu+H_{t}\right)_{k k}|| \Delta L_{j, t} \mid>\epsilon\right), \quad j, k \in\{1,2\}, j \neq k,
$$

depends in this case also on the sign of $\exp \left(\frac{1}{2}\left(\mu+H_{t}(\omega)\right)\right)_{k j}$. To assure that the off-diagonal elements in $\exp \left(\frac{1}{2}(\mu+H)\right)$ are also positive almost surely, we would have to assume that $H$ is positive almost surely. But this seems to be too restrictive.

In the second example we study a bivariate $\operatorname{ECOGARCH}(2,2)$ process. The driving Lévy process $L$ is the same as in the first example. The vectorial state space representation is in this case given by

$$
\begin{aligned}
\operatorname{vech}\left(H_{t}\right) & =\left(I_{3}, 0\right) X_{t} \\
d X_{t} & =\left(\begin{array}{cc}
0 & I_{3} \\
-A_{2} & -A_{1}
\end{array}\right) X_{t} d t+\left(\begin{array}{c}
\tilde{B}_{1} \\
\tilde{B}_{2}
\end{array}\right) d M_{t}
\end{aligned}
$$


with

$$
\begin{gathered}
-A_{1}=\left(\begin{array}{ccc}
-1.0890 & 1.3086 & 0.2193 \\
-1.2412 & -0.6910 & 0.1966 \\
-1.7537 & -0.6331 & -0.4548
\end{array}\right), \quad-A_{2}=\left(\begin{array}{ccc}
0.0466 & -0.5511 & 0.3881 \\
0.2271 & -1.6854 & 0.7785 \\
-0.9972 & 0.9893 & 0.0554
\end{array}\right) \\
\tilde{B}_{1}=B_{0}=I_{3} \\
\tilde{B}_{2}=-A_{1} B_{0}+B_{1}=\left(\begin{array}{ccc}
-0.0890 & 1.3086 & 0.2193 \\
-1.2412 & 0.3090 & 0.1966 \\
-1.7537 & -0.6331 & 0.5452
\end{array}\right)
\end{gathered}
$$

The remaining parameters are chosen as for the ECOGARCH $(1,1)$ process. In Figure 3 we see again parts of the trajectories of the bivariate log-price $G$ as well as the diagonal elements of the volatility process $\exp (\mu+H)$.
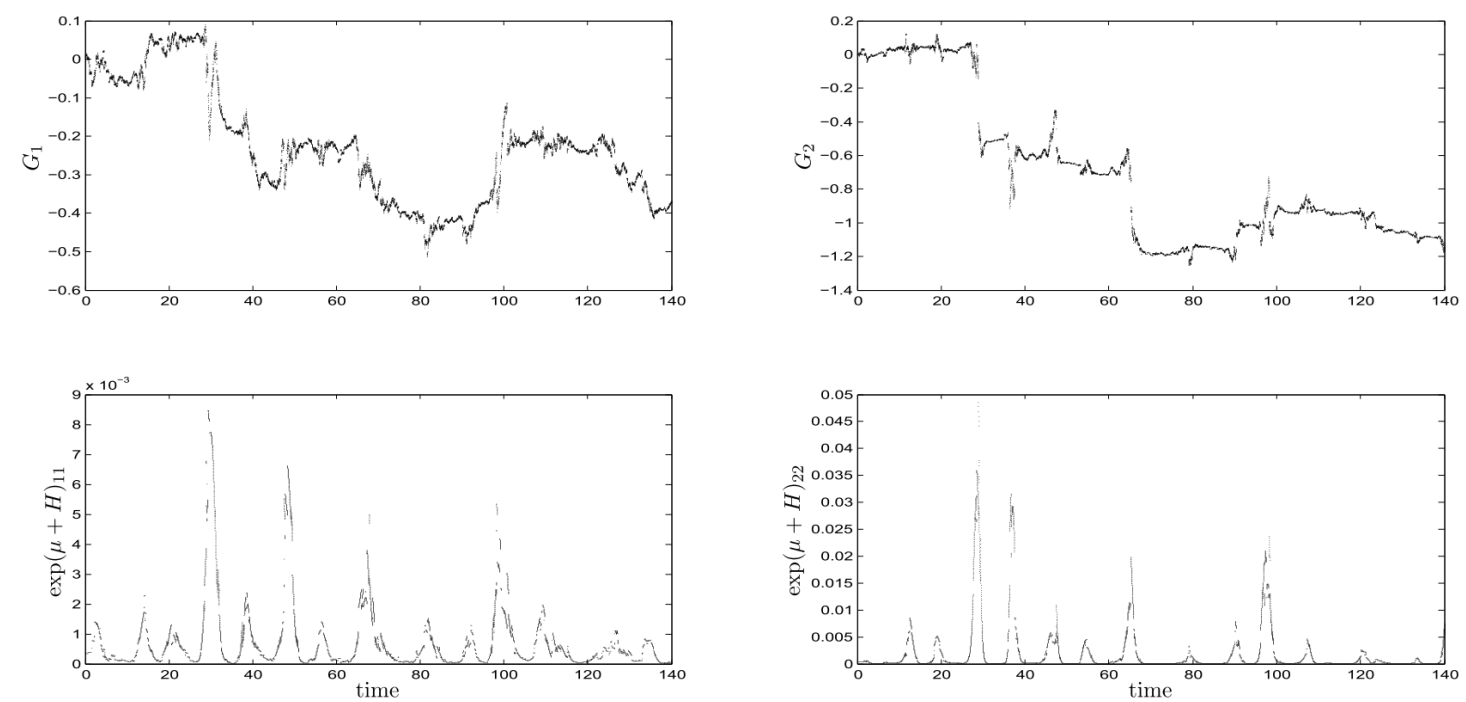

Figure 3: Simulated trajectories of the log-price process $G$ in the top row and the diagonal elements of the volatility process $\exp (\mu+H)$ in the bottom row.

As in the first example we would again expect future volatilities to be negatively correlated with current jumps in the price. To check this assumption we estimated again (4.19) from 1000 simulated trajectories and the average values are

$$
\begin{aligned}
& \widehat{\operatorname{Corr}}\left(\Delta G_{1, t^{1, *},} \exp \left(\mu+H_{t^{1, *}}\right)_{11}\right)=-0.2018 \\
& \widehat{\operatorname{Corr}}\left(\Delta G_{2, t^{2, *},} \exp \left(\mu+H_{t^{2, *}}\right)_{22}\right)=-0.2243 \text {. }
\end{aligned}
$$

The corresponding empirical standard errors are 0.0074 and 0.0116 , respectively. We see again a negative correlation between current returns and future volatility. The negative correlation between jumps in the log-price and the future volatility can also be seen from the plots in Figure 3.

As a third example we consider again the ECOGARCH $(1,1)$ process of the first example. The only differences are the matrices $\Theta$ and $\Gamma$. Now they are chosen in such a way that we will have a positive correlation between current returns and future volatility. In particular they are given by

$$
\Theta=\left(\begin{array}{ll}
0.40 & 0.40 \\
0.01 & 0.01 \\
0.40 & 0.40
\end{array}\right) \quad \text { and } \quad \Gamma=\left(\begin{array}{ccc}
0.01 & 0 & 0 \\
0 & 0.10 & 0 \\
0 & 0 & 0.01
\end{array}\right)
$$


Averaging again over 1000 simulations we get the following empirical correlations

$$
\begin{aligned}
& \widehat{\operatorname{Corr}}\left(\Delta G_{1, t^{1, *},} \exp \left(\mu+H_{t^{1, *}}\right)_{11}\right)=0.3238 \\
& \widehat{\operatorname{Corr}}\left(\Delta G_{2, t^{2, *},} \exp \left(\mu+H_{t^{2, *}}\right)_{22}\right)=0.2921 .
\end{aligned}
$$

The corresponding empirical standard errors are 0.0073 and 0.00067 , respectively, which shows that this is an example for the non-leverage case. Sample trajectories for one of the simulations are shown in Figure 4.
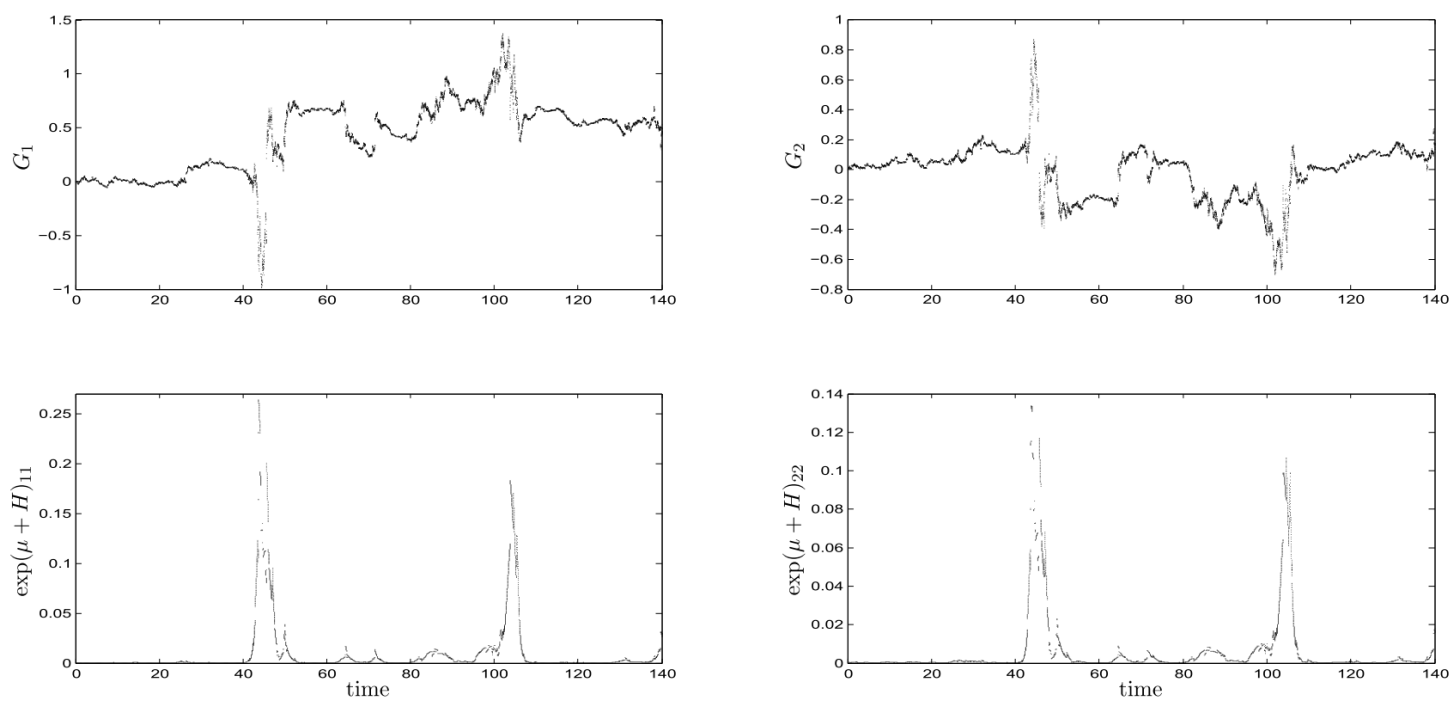

Figure 4: Simulated trajectories of the log-price process $G$ in the top row and the diagonal elements of the volatility process $\exp (\mu+H)$ in the bottom row.

\section{Acknowledgements}

The authors are grateful to the editor and two anonymous referees, Claudia Klüppelberg and Jean Jacod for their very good comments and helpful remarks on a draft of this paper. The second author acknowledges financial support from the Deutsche Forschungsgemeinschaft through the graduate programme "Angewandte Algorithmische Mathematik" at the Technische Universität München during the initial work on the contents of this paper.

\section{References}

[1] Applebaum, D. (2004). Lévy Processes and Stochastic Calculus. Cambridge University Press, Cambridge.

[2] Barndorff-Nielsen, O. and Shepard, N. (2001). Non-Gaussian Ornstein-Uhlenbeck-based models and some of their uses in financial econometrics (with discussion). J. R. Stat. Soc. B Statist. Methodol. 63, 167-241.

[3] Bhatia, R (1997). Matrix Analysis, volume 169 of Graduate Texts in Mathematics. Springer, New York.

[4] Bollerslev, T. (1986). Generalized Autoregressive Conditional Heteroskedasticity. J. Econometrics 31, 307-327. 
[5] Brockwell, P.J. and Davis, R.A. (1991). Time Series: Theory and Methods, 2nd ed. Springer, New York.

[6] Brockwell, P.J. and Lindner, A. (2009). Existence and uniqueness of stationary Lévy-driven CARMA processes. Stochastic Process. Appl. 119, 2660-2681.

[7] Chiu, T.Y.M. and Leonard, T. and Tsui, K.-W. (1996). The Matrix-Logarithmic Covariance Model. J. Amer. Statist. Assoc. 91, 198-210.

[8] Czado, C. and Haug, S. (2009). An ACD-ECOGARCH(1,1) model. J. Financial Econometrics, doi:10.1093/jjfinec/nbp023.

[9] Davydov, Y.A. (1973). Mixing conditions for Markov chains. Theory Probab. Appl. 18, 312328.

[10] Doukhan, P. (1994). Mixing, Vol. 85 Lecture Notes in Statistics. Springer, New York.

[11] Engle, R.F. (1982). Autoregressive Conditional Heteroskedasticity with estimates of the Variance of United Kingdom Inflation. Econometrica 50, 987-1008.

[12] Gourieroux, C. (2006). Continuous time Wishart processes for stochastic risk. Econometric Rev. 25, 177-217.

[13] Haug, S. and Czado, C. (2007). An exponential continuous time GARCH process. J. Appl. Probab. 44, 960-976.

[14] Horn, R.A. and Johnson, C.R. (1991). Topics in Matrix Analysis. Cambridge University Press, Cambridge.

[15] Jacod, J. and Shiryaev, A.N. (2003). Limit Theorems for Stochastic Processes, 2nd ed. Springer, Berlin.

[16] Kawakatsu, H. (2006). Matrix exponential GARCH. J. Econometrics 134, 95-128.

[17] Klüppelberg, C., Lindner, A. and Maller, R.A. (2004). A continuous-time GARCH process driven by a Lévy process: stationarity and second-order behaviour. J. Appl. Probab. 41, 601-622.

[18] Kurtz, T. and Protter, P. (1996). Weak convergence of stochastic integrals and differential equations, in D. Talay and L. Tularo (eds), Probabilistic Models for Nonlinear Partial Differential Equations, Vol. 1627 of Lecture Notes in Mathematics 1-41.

[19] Maller, R.A., Müller, G. and Szimayer, A. (2008). GARCH modelling in continuous time for irregularly spaced time series data. Bernoulli 14, 519-542.

[20] Marquardt, T. and Stelzer, R. (2007). Multivariate CARMA processes. Stochastic Process. Appl. 117, 96-120.

[21] Nelson, D.B. (1990). ARCH models as diffusion approximations. J. Econometrics 45, 7-38.

[22] Nelson, D.B. (1991). Conditional heteroskedasticity in asset returns: a new approach. Econometrica 59, 347-370.

[23] Östermark, R. (2001). Genetic modelling of multivariate EGARCHX-processes: evidence on the international asset return signal response mechanism. Comput. Statist. Data Anal. $38,71-93$. 
[24] Pigorsch, Ch. and Stelzer, R. (2009). A multivariate Ornstein-Uhlenbeck type stochastic volatility model. Submitted for publication.

[25] Protter, P. (2004). Stochastic Integration and Differential Equations, 2nd ed. Springer, New York

[26] Rowland, T. and Weisstein, E.W. (2009) Matrix Exponential. From MathWorld-A Wolfram Web Resource. http:/ / mathworld.wolfram.com/MatrixExponential.html.

[27] Sato, K. (1999). Lévy Processes and Infinitely Divisible Distributions. Cambridge University Press, Cambridge.

[28] Semeraro, P. (2008). A multivariate variance Gamma model for financial applications. Int. J. Theor. Appl. Finance 11, 1-18.

[29] Stelzer, R. (2009). First jump approximation of a Lévy driven SDE process and an application to multivariate ECOGARCH processes. Stochastic Process. Appl. 119, 1932-1951.

[30] Stelzer, R. (2009). Multivariate COGARCH(1,1) processes. Bernoulli 16, 80-115.

[31] Tse, Y. and Hackard, J.C. (2004). Can Island provide liquidity and price discovery in the dark? Review Quant. Finance and Accounting 23, 149-166.

[32] Yang, S.-Y. and Doong, S.-C. (2004). Price and Volatility Spillovers between Stock prices and Exchange Rates: Empirical Evidence from the G-7 Countries. Int. J. Business and Economics 3, 139-153.

Stephan Haug

Zentrum Mathematik

Technische Universität München

Boltzmannstraße 3

D-85748 Garching, Germany

haug@tum.de
Robert Stelzer

TUM Institute for Advanced Study

Technische Universität München

Boltzmannstraße 3

D-85748 Garching, Germany 